Check for updates

Cite this: RSC Adv., 2018, 8, 18519

Received 25th March 2018

Accepted 14th May 2018

DOI: $10.1039 / \mathrm{c} 8 \mathrm{ra02582g}$

rsc.li/rsc-advances

\section{Morphologically controlled synthesis of ionic cesium iodide colloidal nanocrystals and electron beam-induced transformations $\uparrow$}

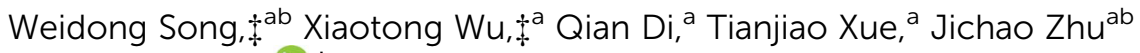 \\ and Zewei Quan (iD *a
}

Colloidal nanocrystals (NCs) have become an important group of novel materials with applications in various fields such as lighting, medicine, and optoelectronic devices. Compared to common semiconductor NCs (e.g., CdSe, CdS, PbS) with covalent bonds and metal NCs (e.g., Au, Ag, Pt) with metallic bonds, the synthesis of colloidal NCs with ionic bonds has rarely been explored, possibly due to their high solubility in polar solvents. In this work, we demonstrate a wet chemical synthesis route to prepare ionic cesium iodide (Csl) colloidal NCs, and they can be controllably made into different morphologies with good uniformity, including nanospheres, hexagonal nanoplates and nanocubes. The degradation of CsI NCs during transmission electron microscopy (TEM) has been investigated, revealing their sensitivity to high energy electron beam irradiation. The as-prepared CsI NCs exhibit strong absorption bands peaking at 275-280 nm, which should be ascribed to the presence of F-centers inside the band gap of CsI NCs. This study provides an efficient way to achieve controllable synthesis of highquality CsI NCs that may find promising applications as advanced nanoscintillators in medical imaging, particle physics, position emission tomography and other various fields.

\section{Introduction}

Nanocrystals (NCs) have attracted a great deal of attention from researchers in various areas for both their fundamental sizeand shape-dependent properties and their many promising technological applications. ${ }^{1-3}$ A quantitative understanding of these important NCs laws has emerged as a direct result of synthetic advances that permit the preparation of monodisperse crystallites of diverse nanoscale size and shape. Currently, most syntheses of NC colloids are limited to semiconducting II-VI, IV-VI and III-V compounds with covalent bonds and metals with metallic bonds such as noble metals and main group metals. One common feature of these substances is that they are insoluble in polar solvents, enabling the facile synthesis and purification of various colloidal NCs. As for ionic compounds that are readily dissolved in polar solvents, such as water and ethanol, the controlled synthesis of NCs with ionic bonds has met limited success. The development of these ionic

${ }^{a}$ Department of Chemistry, Southern University of Science and Technology (SUSTech), Shenzhen, Guangdong 518055, P. R. China. E-mail: quanzw@sustc.edu.cn

${ }^{b}$ School of Chemistry and Chemical Engineering, Harbin Institute of Technology (HIT), Harbin 150001, P. R. China

$\dagger$ Electronic supplementary information (ESI) available. See DOI: $10.1039 / \mathrm{c} 8 \mathrm{ra02582 \textrm {g }}$

$\ddagger$ These authors contributed equally to this work.
NCs requires a robust synthetic route for rational tuning at nanometer length scales.

Cesium iodide (CsI) is a kind of ionic compound that has been widely used as scintillators in many areas such as X-ray image intensifier tubes, photocathodes, and display devices., ${ }^{\mathbf{4 5}}$ Current studies of CsI scintillators have been mostly focused on monocrystalline CsI columns or polycrystalline CsI films deposited on substrates. ${ }^{6-10}$ The size reduction of CsI from bulk crystals to NCs possibly leads to three-dimensional confinement and more overlap of electron and hole wavefunctions, resulting in optical transitions with higher efficiency and faster rates. ${ }^{11-13}$ Therefore, the use of CsI NCs as nanoscitillators is expected to eliminate the issue of slow response of conventional scintillator detectors, and the excellent solubility of CsI colloidal NCs in organic solvents also enables solutionprocessable devices with low cost. However, the controlled synthesis of high-quality CsI colloidal NCs with tunable morphologies still remains a challenging task, ${ }^{\mathbf{1 4}}$ due to its ionic characteristics as mentioned above. On the other hand, CsI is a kind of electron sensitive material, exhibiting obvious degradation under electron beam irradiation. For example, the grain boundaries (known as "dead areas") of CsI films were reported to present white spots under continuous electron beam irradiation. ${ }^{15,16}$ Such small CsI NCs possess large specific surface area, and thus it is of great significance to explore the electron beaminduced transformations of CsI NCs. 
Herein, we report the morphologically controlled synthesis of ionic CsI NCs with narrow size distribution. These CsI colloidal NCs are prepared by a traditional hot-injection method, and the shapes of CsI NCs can be successfully tuned (nanospheres, hexagonal nanoplates, and nanocubes) by adjusting the reactions parameters such as the precursor ratio and ligand category. Such CsI NCs are ultra-sensitive to the high energy electron beam irradiation, and all types of singlecrystalline nanoparticles finally transform into polycrystalline tiny debris with random orientations. The optical properties of as-prepared CsI NCs were also studied by measuring the UV-Vis absorption spectra, and all of them exhibited strong absorption in the ultraviolet region.

\section{Synthesis}

The solution synthesis of CsI colloidal NCs is based on the hotinjection method that is often adopted to prepare a series of nanomaterials, and the reactions are performed under air-free conditions using standard Schlenk technique. CsI NCs are prepared by reacting cesium oleate (Cs-OA) with germanium iodide $\left(\mathrm{GeI}_{2}\right)$ in a high boiling solvent octadecene (ODE), in the presence of certain coordinating ligands. By manipulating the ratio of $\mathrm{I}^{-}$and $\mathrm{Cs}^{+}$precursors (from 1 to 3 ) and the ligand categories, CsI nanospheres, hexagonal nanoplates and nanocubes were synthesized. As the co-precipitation reaction of $\mathrm{Cs}^{+}$ and $\mathrm{I}^{-}$proceeding, the solution color changes from colorless to fawn and then to white, indicating the formation of CsI NCs. The optimal reaction temperature is $180{ }^{\circ} \mathrm{C}$, as there are no products below this temperature and CsI NCs with irregular shapes appear at higher temperature (Fig. S1†). Detailed experimental procedures are shown below.

\section{Chemicals}

Germanium(II) diiodide ( $\mathrm{GeI}_{2} \geq 99.8 \%$ trace metals basis), cesium carbonate $\left(\mathrm{Cs}_{2} \mathrm{CO}_{3}\right.$, reagent plus, 99\%), 1-octadecene (ODE, technical grade, 90\%), oleylamine (OAm, 70\%), oleic acid (OA, 90\%), tri- $n$-octylphosphine (TOP, 97\%) and hexane (99.9\%), were purchased from Sigma-Aldrich. All chemicals were used without any further purification.

\section{Synthesis of spherical CsI NCs}

$\mathrm{GeI}_{2}$ precursor solution was prepared by dissolving $0.01 \mathrm{~g} \mathrm{GeI}_{2}$ with $2 \mathrm{~mL}$ ODE, $0.2 \mathrm{~mL}$ OA and $0.2 \mathrm{~mL}$ OAm in a vial protected by an Argon gas, which is heated to $100{ }^{\circ} \mathrm{C}$ in an oil bath. ODE (3 $\mathrm{mL})$, OA $(0.1 \mathrm{~mL})$ and $\mathrm{Cs}_{2} \mathrm{CO}_{3}(0.015 \mathrm{~g})$ were loaded into a $100 \mathrm{~mL}$ 3-neck flask and vacuumed at $110{ }^{\circ} \mathrm{C}$ for $1 \mathrm{~h}$ unceasingly. After complete solubilization of $\mathrm{Cs}_{2} \mathrm{CO}_{3}$, the temperature was raised to $180{ }^{\circ} \mathrm{C}$, and then as-prepared $\mathrm{GeI}_{2}$ solution was swiftly injected, and the reaction mixture was cooled down 5 min later by a water bath.

\section{Synthesis of hexagonal CsI NCs}

The same procedure as that for spherical CsI NCs was followed, except that the quantity of the $\mathrm{GeI}_{2}$ was increased to $0.03 \mathrm{~g}$, and the reaction time was increased to 10 minutes.

\section{Synthesis of cubic CsI NCs}

The same procedure as that for hexagonal CsI nanoplates was followed, except that the addition $1 \mathrm{~mL}$ TOP was introduced into the dissolution process of $\mathrm{GeI}_{2}$.

\section{Isolation and purification of CsI NCs}

The crude solution was cooled down with water bath and CsI NCs were collected by centrifugation for $8 \mathrm{~min}$ at $8000 \mathrm{rpm}$. After that, the supernatant was discarded and the precipitates were re-dispersed in $5 \mathrm{~mL}$ hexane and centrifuged again for $8 \mathrm{~min}$ at $8000 \mathrm{rpm}$. After repeating this step one more time, the precipitates were re-dispersed in $5 \mathrm{~mL}$ hexane for storage.

\section{Post-synthetic treatment of CsI NCs with ethanol}

The as-purified cubic CsI NC colloidal NCs was treated with 20 $\mu \mathrm{L}$ ethanol first, and then $5 \mathrm{~mL}$ hexane was added and centrifuged again for $8 \mathrm{~min}$ at $8000 \mathrm{rpm}$. The precipitates were finally re-dispersed in $5 \mathrm{~mL}$ hexane for characterizations.

\section{Synthesis of CsI NCs with different precursors}

The same procedure as that for CsI NCs with $\mathrm{GeI}_{2}$ as $\mathrm{I}^{-}$ precursor was followed, except that the $\mathrm{GeI}_{2}$ was replaced by $\mathrm{NaI}, \mathrm{KI}$ and $\mathrm{CaI}_{2}$, respectively.

\section{Characterization techniques}

Powder X-ray diffraction (XRD) patterns were acquired using a Rigak Smartlab with $\mathrm{Cu} \mathrm{K} \alpha$ operating at $45 \mathrm{kV} / 20 \mathrm{~mA}$. The solutions are dropped on amorphous silicon wafer to form windstopper membrane for testing. TEM images were recorded using Tecnai F30 microscope operated at $300 \mathrm{kV}$. UV-Vis absorption spectra for colloidal solutions were collected using a Cary 5000 Series UV-Vis-NIR Spectrophotometer.

\section{Results and discussion}

The morphology and crystallinity of spherical CsI NCs are illustrated in Fig. 1. Fig. 1a shows the monodisperse CsI nanoparticles with an average diameter of $15 \mathrm{~nm}$, and these uniform nanospheres exhibit the self-assembly phenomenon to form 2D hexagonal packing pattern. A group of well-defined crystal lattices are observed in the HRTEM image, and the interplane distance of $3.25 \AA$ corresponds to the $\{110\}$ crystal plane of CsI in Pm3m phase (inset of Fig. 1b). It is interesting to find that as CsI spherical NCs are exposed to electron beam irradiation for a short period (several seconds), the single crystallinity can be well maintained, as shown in the selectedarea electron diffraction (SAED) pattern (Fig. 1c). When electron beam irradiation accumulates to tens of seconds, the surface inhomogeneity of CsI nanosphere begins to appear, as seen in the inset of Fig. 1d. The corresponding SAED pattern of one nanosphere (Fig. 1d) after sufficient beam irradiation exhibits a polycrystalline state, confirming the transformation of CsI nanospheres from single crystal to small pieces with random orientations. The sensitivity of CsI to electron beam has 

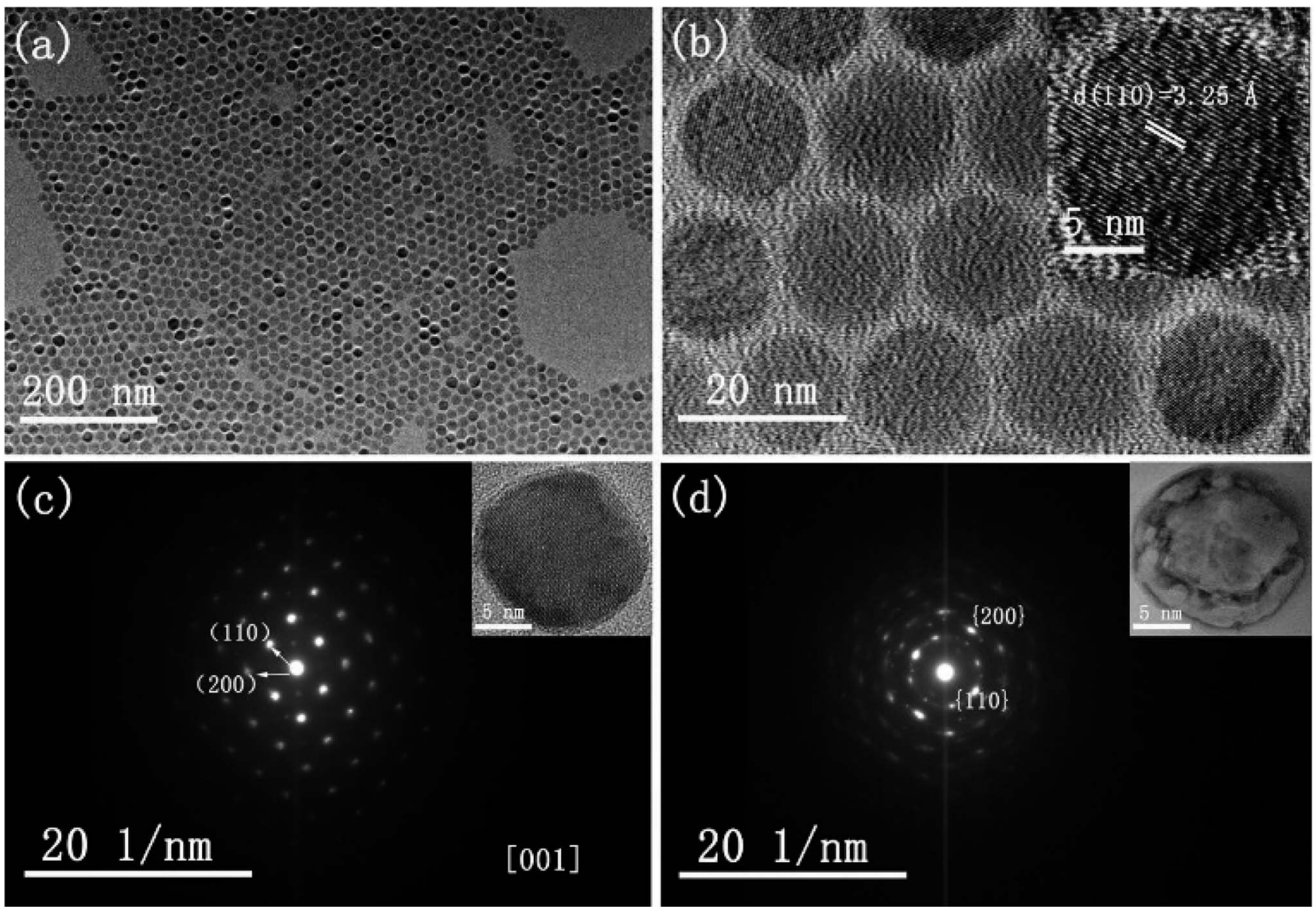

Fig. 1 Monodisperse CsI nanospheres and their structural characterizations. TEM images of (a) non-close packed 2D stratiform superlattice (b) HRTEM image of CsI nanospheres. SAED patterns of a single CsI nanosphere under electron beam irradiation for several seconds (c) and tens of seconds (d). Insets of (c) and (d) are TEM images of the corresponding CsI nanosphere.

been discovered in previous studies. ${ }^{16-19}$ The specific surface area is increased with reducing particle size,${ }^{\mathbf{2 0}}$ hence, these asprepared CsI NCs should be highly sensitive to electron beam irradiation. Under electron beam irradiation, there are two main specimen-electron interactions that are known as knockon damage and ionization radiolysis. ${ }^{21}$ For radiolysis damage, the energy of incident electrons stores in an exciton, and the relaxation time for the exciton should be long enough so that mechanical relaxation of the atoms can cause bonding instabilities. Therefore, crystallinity change or element loss will occur in this case. As for knock-on damage, an incident energetic electron transfers a significant amount of energy to the atoms of the specimen in a direct collision way, which is sufficient to remove an atom from its site or sputter it from the surface. For high-energy electron beam (300 kV), knock-on based sputtering of the material will dominate. ${ }^{20-22}$ Similar to ionic $\mathrm{NaYF}_{4} \mathrm{NCs}^{23}$ the knock-on damage should be the dominant mechanism of damage to CsI NCs based on our experimental observations, because there is no element loss in the electron irradiation process. Furthermore, these uniform CsI nanospheres are rapidly transformed into other shapes when 20 $\mu \mathrm{L}$ ethanol is added into CsI NCs colloidal solution, as shown in Fig. S2.† Such changes are due to the ionic nature of CsI, resulting in the dissolution of CsI nanospheres in ethanol and subsequent formation of irregular shapes.

The shape-controlled synthesis of CsI colloidal NCs can also be realized with delicate adjustment of experiment parameters. Uniform hexagonal CsI nanoplates were obtained by increasing the amount of $\mathrm{I}^{-}$precursor and increasing the reaction time, while other conditions are kept the same as in the synthesis of CsI nanospheres (see synthesis section for details). Fig. 2a shows the typical TEM image of the CsI hexagonal nanoplates with good monodispersity and sharp edges, and the average length of the nanoplates is about $40 \mathrm{~nm}$. Interestingly, these uniform hexagonal CsI nanoplates can self-assemble into ordered hexagonal packing, the behavior of anisotropic NCs assembled supercrystals has been investigated deeply for years, which may emerge novel properties. ${ }^{24-27}$ The HRTEM image of the hexagonal CsI nanoplate shows well-resolved lattice fringes in the inset of Fig. 2a. The interlayer distance of $3.28 \AA$ is consistent with the (110) lattice spacing is nanospheres mentioned above, these CsI hexagonal observed over the entire nanoplate surface. Similar to the nanoplates undergo obvious electron beam irradiation-induced changes, and these individual single crystalline nanoplates finally transform into connected hexagonal net structures (Fig. 2b), due to the movement 

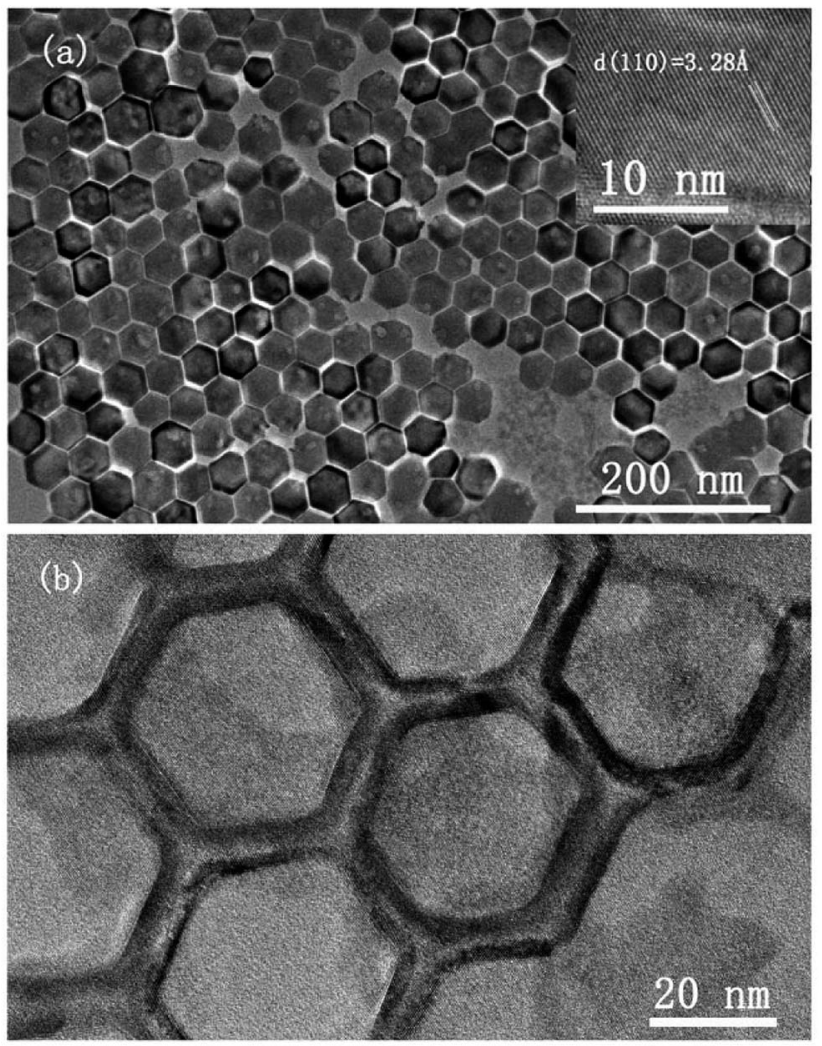

Fig. 2 TEM images of hexagonal CsI nanoplates under electron beam irradiation for (a) several seconds and (b) tens of seconds. Inset of (a) shows lattice fringes of CsI nanoplates in HRTEM image.

of partial CsI species from surface center to interparticle region under continuous electron irradiation.

Furthermore, the shape of CsI NCs can be changed from hexagonal nanoplates to nanocubes, when TOP solvent is additionally introduced into $\mathrm{I}^{-}$precursor solution and the reaction time is also increased to 10 minutes. Fig. 3a shows the TEM image of as-prepared CsI nanocubes with an average side length of $40 \mathrm{~nm}$. The lattice fringes shown in the inset of Fig. 3a show the TEM image of as-prepared CSI nanocubes with average edge length of $40 \mathrm{~nm}$. The lattice fringes shown in the inset of Fig. 3a indicate the high crystallinity of these nanocubes, and the $3.25 \AA$ lattice spacing also corresponds to $\{110\}$ planes of CsI in $P m 3 m$ phase. Under electron beam irradiation, these single crystalline CsI nanocubes are gradually collapsed and part of CsI are diffused to interparticle regions (Fig. $3 \mathrm{~b}$ ).
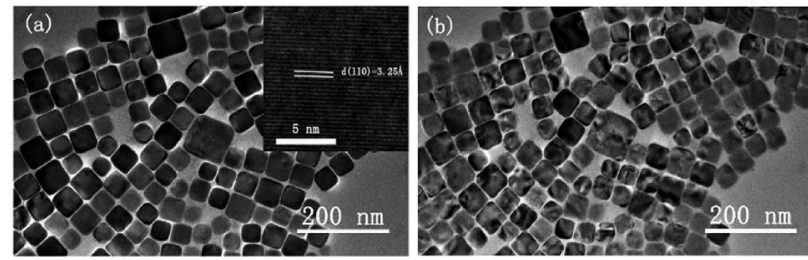

Fig. 3 Monodisperse CsI nanocubes and their structural characterizations. TEM images of CsI nanocubes under electron beam irradiation for (a) several seconds and (b) tens of seconds. Inset of (a) shows lattice fringes of $\mathrm{Csl}$ nanocubes.
To further analyze the crystalline structure, XRD patterns were used to characterize these samples. It is known that CsI can crystallize in hexagonal, tetragonal or cubic phases, and all as-prepared CsI NCs in this work are found to crystallize in cubic phase with $P m \overline{3} m$ space group (PDF \#06-0311). As shown in Fig. 4, peaks at $2 \theta=27.59^{\circ}, 39.42^{\circ}, 48.79^{\circ}$ and $56.97^{\circ}$ correspond to diffractions from (110), (200), (211) and (220) crystal planes, respectively. No extra peaks are detected in these XRD patterns, demonstrating the phase purity of as-prepared CsI NCs. Although as-prepared CsI NCs have different shapes (nanospheres, hexagonal nanoplates, and nanocubes), the strongest diffraction peaks in all these samples are (110) peak, which is consistent with the exclusive presence of (110) lattice planes in HRTEM images (Fig. 1-3).

The controlled synthesis of colloidal NCs with varied morphologies has been systematically investigated in different systems including metal (e.g., $\mathrm{Au}, \mathrm{Ag}, \mathrm{Pt})$, semiconductor (e.g., $\mathrm{CdSe}, \mathrm{PbS}, \mathrm{InP})$, and even insulators (e.g., $\left.\mathrm{NaYF}_{4}\right)$. It is known that experimental conditions such as the type and ratio of precursors and ligand types, reaction temperature and time, play important roles in determining the final shape of asprepared NCs. ${ }^{28-30}$ The illustration of the shape-controlled preparation of CsI NCs is presented in Scheme 1, which indicates that these morphology variations in this work are attributed to the different precursor ratio and the choice of coordinating ligands. As the ratio of $\mathrm{I}^{-}$and $\mathrm{Cs}^{+}$precursors is increased from 1 to 3 , the shape of CsI NCs changes accordingly from nanospheres to hexagonal nanoplates. The formation of isotropic nanospheres is due to the comparable growth rate along different directions. Based on the Fast Fourier Transform (FFT) analysis, the nanoplate surface that is perpendicular to the incident electron beam is indexed to be $\{111\}$ planes (Fig. S3b†). It is reasonable to conclude that the presence of more $\mathrm{I}^{-}$precursor during the synthesis slows down the growth of $\{111\}$ planes, and therefore $\{111\}$ planes are finally preserved on CsI NCs to form anisotropic hexagonal nanoplates. As additional TOP ligand is introduced into the reaction, the morphology of CsI NCs can be further modified from hexagonal

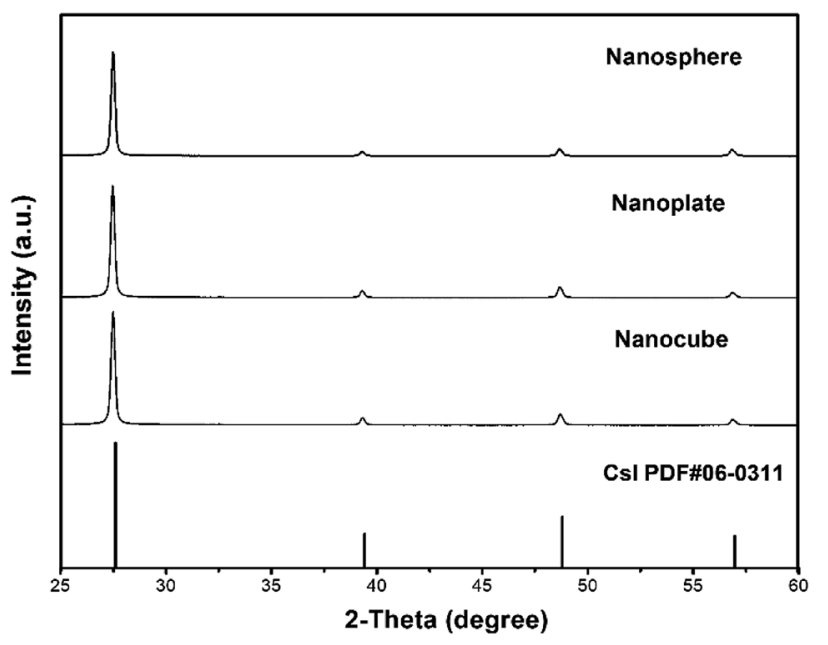

Fig. 4 XRD patterns of CsI NCs with different morphologies. 

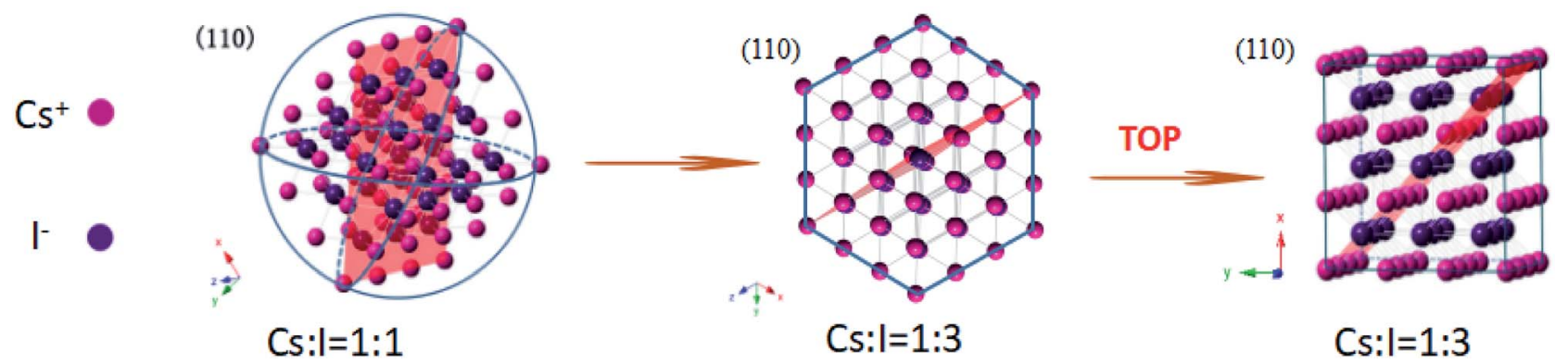

Scheme 1 Schematic illustration of the formation of CsI NCs with different shapes.

nanoplates to nanocubes that are enclosed by six $\{100\}$ planes, as confirmed by FFT analysis (Fig. S3c $\dagger$ ). This is due to the selective coordination of TOP ligand onto $\{100\}$ planes, and thus the growth of $\{100\}$ planes are obviously slowed down to produce CsI nanocubes. ${ }^{31,32}$ It should be noted that similar phenomena have been commonly observed in other nanoparticle systems, ${ }^{33,34}$ however, the underlying mechanism of shape-controlled synthesis of CsI NCs is still not clear yet, which requires further in-depth investigations.

In this work, we have controllably synthesized the CsI NCs with different morphologies, and the effect of different $\mathrm{I}^{-}$ precursors on the formation of CsI NCs has also been studied by replacing $\mathrm{GeI}_{2}$ with other low-cost precursors, such as NaI, KI and $\mathrm{CaI}_{2}$. When NaI, $\mathrm{KI}$ or $\mathrm{CaI}_{2}$ was used as the $\mathrm{I}^{-}$precursor (Fig. S4 $\dagger$ ), both the shape uniformity and size distribution are not comparable to the CsI NCs that are synthesized by $\mathrm{GeI}_{2}$, although a series of reaction conditions have been tried on each precursor. These control experiments reveal the unique role of $\mathrm{GeI}_{2}$ precursor in the shape-controlled synthesis of high-quality CsI NCs. This is probably due to the stronger affinity of $\mathrm{Ge}^{2+}$ and $\mathrm{I}^{-}$in $\mathrm{GeI}_{2}$ compared to other precursors, controlling the release of $\mathrm{I}^{-}$during the synthesis of CsI NCs.

Fig. 5 shows the UV-Vis absorption spectra of as-prepared CsI NCs, which reveals the sharp absorption edge at 275-280 nm. Such absorption should be attributed to the presence of F-

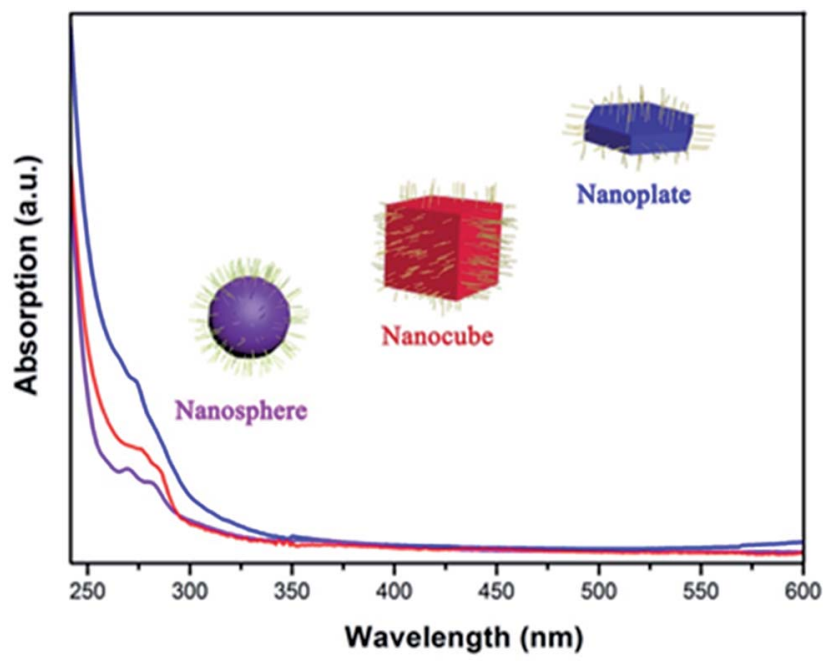

Fig. 5 UV-Vis absorption spectra of these as-prepared CsI NCs with different shapes. centers inside the band gap of CsI NCs, and this value is comparable to those in previous researches. ${ }^{35,36}$ Bulk CsI is essentially insulator with large band gap $(6.4 \mathrm{eV})$, and it is thus difficult to observe band gap absorption longer than $200 \mathrm{~nm}^{37}$ In classical theories, for an alkali atom, one $\Gamma_{4}$ orbital triplet occurs in the excited state of the F-band transition, and the spin-orbit interaction splits the levels into a doubly degenerate $\Gamma_{6}$ and a quadruply degenerate $\Gamma_{8}$ state for cubic symmetry crystal. On the other hand, the instantaneous environment of the F-center is related to lattice vibrations that may lead to the dynamic distortion of surrounding ions, and this may also influence the absorption intensity of CsI NCs with diverse morphologies. ${ }^{38-43}$ The optical absorptions in these CsI NCs are most likely due to this low energy state of the F-center exciton, and the dynamic distortion of these tiny NCs may also contribute to some extent. ${ }^{38}$

\section{Conclusions}

In summary, a wet chemical approach has been developed for the shape-controlled synthesis of high-quality colloidal CsI NCs with ionic bonds, including nanospheres, hexagonal nanoplates and nanocubes. All these CsI nanoparticles are sensitive to high energy electron beam, and obviously degrade under continuous electron beam irradiation. These as-prepared CsI NCs exhibit strong absorption bands peaking at $275-280 \mathrm{~nm}$, due to the presence of F-centers within band gap. This work provides a novel way to synthesize ionic CsI NCs, and these investigations may enable their application in different fields such as nanoscintillator.

\section{Conflicts of interest}

There are no conflicts to declare.

\section{Acknowledgements}

This work is supported by the National Natural Science Foundation of China (NSFC) (No. 51772142), Shenzhen Science and Technology Innovation Committee including fundamental research projects (No. JCYJ20170412152528921, JCYJ20160530190842589), Peacock Team (KQTD2016053019134356) and Peacock Technology Innovation Project (KQJSCX20170328155428476), Development and Reform Commission of Shenzhen Municipality (Novel 
Nanomaterial Discipline Construction Plan), and start-up fund and Presidential fund from SUSTech. This work was also supported by the Pico Center at SUSTech that receives support from Presidential fund and Development and Reform Commission of Shenzhen Municipality.

\section{References}

1 H. Wang, B. Zeng, Y. Zhao, M. Li, H. Liu and T. Huang, J. Nanosci. Nanotechnol., 2018, 18, 730-734.

2 S. Sun, D. Yuan, Y. Xu, A. Wang and Z. Deng, ACS Nano, 2016, 10, 3648-3657.

3 Q. Shi, D. Dong, K. J. Si, D. Sikdar, L. W. Yap, M. Premaratne and W. Cheng, ACS Nano, 2018, 2, 1014-1022.

4 D. Shiffler, M. Lacour, K. Golby and M. Sena, IEEE Trans. Plasma Sci., 2001, 29, 445-451.

5 J. A. Rowlands and K. W. Taylor, Med. Phys., 1983, 10, 786795.

6 C. Y. Chen, S. H. Chen, C. C. Chen and J. S. Lin, Mater. Lett., 2015, 148, 138-141.

7 M. Strotzer, M. Völk, T. Wild, L. P. Von and S. Feuerbach, Radiology, 2000, 215, 512-515.

8 X. Rong, C. Shaw, X. Liu, M. Lemacks and S. Thompson, Med. Phys., 2001, 28, 2328-2335.

9 W. C. Barber, N. E. Hartsough and J. S. Iwanczyk, IEEE Trans. Nucl. Sci., 2009, 56, 1012-1016.

10 T. Okamura, S. Tanaka, K. Koyama, N. Norihumi, H. Daikokuya, T. Matsuoka, K. Kishimoto, M. Hatagawa, H. Kudoh and R. Yamada, European Radiology, 2002, 12, 1741-1747.

11 C. W. Hun, P. C. Chen, K. J. Huang and C. C. Chen, Thermosense: Thermal Infrared Applications XXXVII, 2015, p. $94850 \mathrm{M}$.

12 N. Y. Morgan, G. Kramermarek, P. D. Smith, K. Camphausen and J. Capala, Radiat. Res., 2009, 171, 236-244.

13 J. Martin, A. D. Scaccabarozzi, A. Nogales, R. Li, D. M. Smilgies and N. Stingelin, Eur. Polym. J., 2016, 81, 650-660.

14 J. Shamsi, Z. Dang, P. Ijaz, A. L. Abdelhady, G. Bertoni, I. Moreels and L. Manna, Chem. Mater., 2018, 30, 79-83.

15 B. Liang, L. G. Shuang Liu, D. Chen, Y. Liu, Z. Zhong and L. Xiong, J. Phys.: Conf. Ser., 2016, 680, 012022.

16 A. S. Tremsin, S. Ruvimov and O. H. W. Siegmund, Nucl. Instrum. Methods Phys. Res., 2000, 447, 614-618.

17 K. Konashi, M. Yamawaki and T. Yoneoka, J. Nucl. Mater., 1988, 160, 75-80.

18 X. Duan, T. Kim, L. Han, J. Ma, X. Du and W. Zheng, Sci. Rep., 2013, 3, 3218.

19 Triloki, B. Dutta and B. K. Singh, Nucl. Instrum. Methods Phys. Res., 2012, 695, 279-282.
20 D. V. Talapin, J. S. Lee, M. V. Kovalenko and E. V. Shevchenko, Chem. Rev., 2010, 110, 389-458.

21 O. Ugurlu, J. Haus, A. A. Gunawan, M. G. Thomas, S. Maheshwari, M. Tsapatsis and K. A. Mkhoyan, Phys. Rev. $B, 2011,83,1127-1134$.

22 J. Kotakoski, C. H. Jin, O. Lehtinen, K. Suenaga and A. V. Krasheninnikov, Phys. Rev. B, 2010, 82, 113404.

23 X. Sun, B. Wang, I. Kempson, C. Liu, Y. Hou and M. Gao, Small, 2014, 10, 4711-4717.

24 R. Li, J. Zhang, R. Tan, F. Gerdes, Z. Luo, H. Xu, J. A. Hollingsworth, C. Klinke, O. Chen and Z. Wang, Nano Lett., 2016, 16, 2792-2799.

25 M. Liu, Z. Wang, J. Liu, G. Wei, J. Du, Y. Li, C. An and J. Zhang, J. Mater. Chem. A, 2017, 5, 1035-1042.

26 J. Zhang, J. Zhu, R. Li, J. Fang and Z. Wang, Nano Lett., 2017, 17, 362-367.

27 Z. Wang, K. Bian, Y. Nagaoka, H. Fan and Y. C. Cao, J. Am. Chem. Soc., 2017, 139, 14476-14482.

28 B. Liu, X. Deng, Z. Xie, Z. Cheng, P. Yang and J. Lin, Adv. Mater., 2017, 29, 1604878.

29 Z. Xie, X. Deng, B. Liu, S. Huang, P. Ma, Z. Hou, Z. Cheng, J. Lin and S. Luan, ACS Appl. Mater. Interfaces, 2017, 9, 30414-30425.

30 Y. Shi, X. Deng, S. Bao, B. Liu, B. Liu, P. Ma, Z. Cheng, M. Pang and J. Lin, Chem.-Asian J., 2017, 12, 2183-2188.

31 H. S. Chen and R. V. Kumar, J. Phys. Chem. C, 2009, 113, 12236-12242.

32 C. B. Murray, D. J. Norris and M. G. Bawendi, J. Am. Chem. Soc., 1993, 115, 8706-8715.

33 B. Ding, C. Yu, C. Li, X. Deng, J. Ding, Z. Cheng, B. Xing, P. Ma and J. Lin, Nanoscale, 2017, 9, 16937-16949.

34 L. Feng, F. He, Y. Dai, S. Gai, C. Zhong, C. Li and P. Yang, Biomater. Sci., 2017, 5, 2456-2467.

35 A. Farzaneh, M. R. Abdi, K. R. E. Saraee, M. Mostajaboddavati and A. Quaranta, Opt. Mater., 2016, 55, 22-26.

36 A. Farzaneh, M. R. Abdi, K. R. E. Saraee, M. Mostajabaldaavati and A. Quaranta, J. Sol-Gel Sci. Technol., 2016, 78, 313-321.

37 E. Knittle and R. Jeanloz, Science, 1984, 223, 53-56.

38 P. R. Moran, Phys. Rev., 1965, 137, A1016-A1027.

39 U. Rössler, Phys. Rev., 1965, 137, A1016-A1027.

40 K. I. Said and G. W. Green, J. Phys. C: Solid State Phys., 2001, 10, 479-488.

41 R. Monnier, K. S. Song and A. M. Stoneham, J. Phys. C: Solid State Phys., 1977, 10, 4441-4448.

42 C. C. Klick, D. A. Patterson and R. S. Knox, Phys. Rev., 1964, 133, 1717-1723.

43 A. D. Brothers and D. W. Lynch, Phys. Rev., 1967, 164, 11241135. 